\title{
Research of extractors for the extraction of target components from plant materials of various internal structures
}

\author{
Maksim Prosin ${ }^{1 *}$, Dmitrii Borodulin ${ }^{1}$, Elena Safonova ${ }^{1}$, and Yana Golovacheva ${ }^{1}$ \\ ${ }^{1}$ Institute of Engineering Technologies, Kemerovo State University, 6, Krasnaya St., Kemerovo, \\ 650000, Russia
}

\begin{abstract}
Manufacturers prefer berries and fruits, but there are also other products made with seeds, aromatic and medicinal herbs, spices, as well as other components. The main production stage for obtaining tinctures and aromatic alcohols is the extraction process. Currently, a large number of designs of extractors for solid-liquid system of periodic and continuous action have been developed. All of them are different from each other in terms of efficiency, energy consumption and applicability in a particular production. The purpose of this work is to study and analyze the influence of the structure of raw materials on the efficiency of extraction in equipment of various types. To carry out the research, three types of raw materials were selected from various groups: rose hips, penny root and chaga mushrooms. The most suitable and promising extractor designs are the Soxlhet extractor; centrifugal extractor; rotary-pulsating apparatus. The results of the work, it was concluded that the most versatile apparatus is the rotary-pulsating apparatus, since during its operation the raw material is crushed. Plant raw materials give the most complete target components with list time. In devices of similar designs, the different internal structure of raw materials does not affect the quality of the extracts obtained.
\end{abstract}

\section{Introduction}

Many centuries have passed since the discovery of alcohol, but still the demand for this product has not decreased. The market for alcoholic beverages has been filled with various types and sorts, from classic inexpensive sorts to premium drinks.

If the French could boast of wine, the Portuguese - port, the Scots - whiskey, and then the Russians became famous for their variety of tinctures. For a long time, everything that can ferment has been fermented. In terms of the number of varieties of tinctures, our country has no equal. Tincture is an alcoholic beverage produced by infusing alcohol of different strengths on various ingredients [1].

The main raw material for obtaining tinctures has always been and remains - vegetable raw materials. Modern manufacturers give preference to berries and fruits, but there are

\footnotetext{
* Corresponding author: prosinmv@,yandex.ru
} 
also individual options for products made on seeds, aromatic and medicinal herbs, spices, and other components [2].

In alcoholic beverage production, over 100 types of plant raw materials are used to obtain tinctures. The chemical composition of plant materials is very complex and diverse. Not only do different types of plants differ in their composition, but within the same species, different varieties have a different chemical composition. The content of certain substances fluctuates in wide ranges depending on many reasons. First of all, in this regard, the climate, meteorological conditions of the growing season and soil quality affect. The weather is of great importance. Even the size of the fruits of the same plant affects their chemical composition [3, 4].

Today, in connection with the growth of consumer abilities of the Russian population and an increase in demand for alcoholic beverages, the issues of import substitution are becoming topical. To reduce the cost and expand the linear product range, it is necessary to use the main raw materials that grow in Siberia.

The main production stage for obtaining tinctures and aromatic alcohols is the extraction process. Today, extraction is one of the main processes used in almost all industries. In the food industry, this process is used to a greater extent in the production of many products [3-7].

As for the hardware design of the extraction process, a large number of designs of extractors have been developed for the solid-liquid system of periodic and continuous action. All of them are different from each other in terms of efficiency, energy consumption and applicability in a particular production [3-8].

Increasing the speed of technological processes and the productivity of technological equipment with a simultaneous increase in the quality of the final product are the main goals that designers and developers set themselves when creating new or modernizing existing designs of devices and machines. Therefore, the research and development of new equipment for efficient processes is an urgent task of technical sciences.

In our opinion, it is necessary to study the effect of equipment on the rate of extraction during the preparation of various extracts from plant materials of various structures, which will make it possible to select rational operating modes in the production of tinctures and identify the most versatile equipment for increasing the variety of products.

The purpose of this work is to study and analyze the influence of the structure of raw materials on the efficiency of extraction in equipment of various types.

In accordance with the purpose of the following tasks:

- investigation of the extraction process using a rotary-pulsating extractor, using a centrifugal extractor and using a Soxlhet extractor;

- investigation of the influence of the internal structure of plant raw materials on the extraction of target components from the root of the penny root, chaga mushroom and rose hips;

- determination of rational operating parameters of the extraction process and selection of the most efficient equipment;

- organoleptic assessment of the obtained extracts.

\section{Materials and methods}

The effect of the structure of a substance on the rate of extraction by various types of extractors was studied using an water-alcohol solution.

A water-alcohol solution based on premium-class ("Luxe") ethyl alcohol of "Luxe", meeting the Russian Standards 5962-2013 was used as the extractant. The premium-class ("Luxe") ethyl alcohol is a grain ethyl alcohol of the highest quality obtained by rectification from wheat, barley, rye grain or a mixture of grains. Most of the vodka and 
liquors produced in Russia are made from premium-class ("Luxe") ethyl alcohol. It is a transparent colorless liquid, without foreign odors and tastes. The content of ethyl alcohol is $96.5 \%$. The water used in research meets the requirements of Russian Standard 6709-72.

To carry out the research, three types of raw materials were selected from different groups: rose hips, penny root and chaga mushrooms. This raw material was selected based on its availability in Siberia, as well as because of the high content of biologically active substances. All raw materials are well preserved in a dried state [4].

Rose hips contain a large amount of vitamin A, C, B1, B2, PP, K, pantothenic acid and flavonoids. It is used for general immunity and increases resistance to adverse factors, has a positive effect on the metabolism in the human body, on the synthesis of hormones and tissue regeneration.

Penny root has a charitable effect on the human body due to the contained catechins, tannins, polysaccharides, vitamin $\mathrm{C}$, some alkaloids, etc. It has a number of useful properties and has a positive effect on cleansing the body, has an anti-inflammatory effect, etc.

The chaga mushroom has the widest chemical composition, which determines the spectrum of its useful properties. In particular, it contains polyphenols with carboxylic acids, agaric acid, resins and maranium. It is this composition that gives it properties that have a general strengthening effect on the human body and a beneficial effect on the digestive system..

The composition of plant raw materials for the preparation of infusions may vary depending on the time and place of collection, weather conditions prevailing in the season of collection and growing of the plant.

Storage of all selected types of raw materials for research is possible for a long period in a dried state, subject to all requirements for their storage conditions. All of them should be stored in a dry, well-ventilated area to avoid dampness. All raw materials were collected during the period of its greatest saturation with biologically active substances, that is, from the end of August to October. All roots, fruits and mushrooms must be used within a year, otherwise in the future there is a sharp decrease in the content of nutrients.

Currently, a huge variety of equipment is known for carrying out the extraction process in a solid-liquid system. Apparatus designs that are completely independent are considered to be well-proven in the production environment. Where no preliminary preparation of raw materials, its grinding or crushing is required.

In our opinion, the most suitable and promising extractor designs are:

- Soxlhet extractor;

- centrifugal extractor;

- $\quad$ rotary-pulsating apparatus (RPA).

The main advantage of the Soxlhet extractor is that pure alcohol is fed inside the extractor; the alcohol is not saturated with the extracted substance, which contributes to a more effective enrichment with aromatic oils and esters. The Soxlhet extractor is designed for obtaining extracts from various raw materials and fast flavoring of strong drinks.

The classic method of producing tinctures is a time-consuming process, while the Soxlhet extractor allows reducing the time of preparation of tinctures from several weeks, or even months, to several hours. The principle of its operation is based on repeated processing of aromatic raw materials with a small volume of the extracting solvent (a strong alcohol-containing solution), as a result of which the aromatic compounds are dissolved from the raw materials, forming an extract.

The advantages of the Soxlhet extractor are: simple and clear design, production process continuity, ease of visual monitoring of the process, a low flow of solvent and the possibility of its reuse after stripping and distillation. 
Fig. 1 shows the design of the Soxlhet extractor. It consists of a distillation still (1) with a siphon arm (5) installed in it, which has a distillation arm (3) and a siphon (4). The thimble (6), into which the extract raw materials are loaded, is installed in the siphon. The extractor chamber (2) is closed with the thimble cover (7). The reflux condenser (8) is fixed to the extractor chamber with of a connection valve. The cold water for cooling the studied liquid flows in through the inlet nozzle (9), and the used water flows out through the outlet nozzle (10).

When the liquid in the distillation still (1) boils, the alcohol-containing vapour rises through the distillation arm (3), the vapour reaches the reflux condenser (8), it is cooled and turns into the liquid, which drips into the thimble (6) and wets the raw materials loaded in it. As the thimble is filled with the solvent, the aromatic compounds are extracted into the alcohol. After the thimble is completely filled with alcohol, the liquid reaches the upper level of the siphon (4), the thimble chamber is emptied. The solution flows down the siphon arm (5) into the distillation still (1). Depending on the set experimental conditions, this cycle is repeated again. Thus, the extraction process may be repeated many times [9-11].

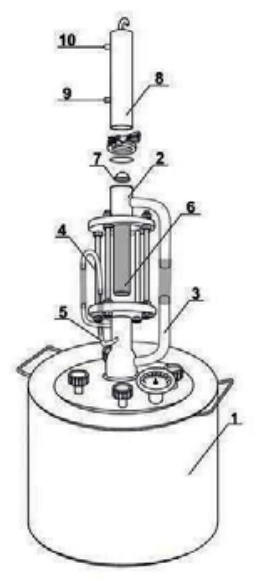

Fig. 1. Soxlhet extractor: 1 - distillation still, 2 - extractor chamber, 3 - distillation arm, 4 - siphon, 5 - siphon arm, 6 - thimble, 7 - thimble cover, 8 - reflux condenser, 9 - cooler inlet nozzle, 10 - cooler outlet nozzle.

Rotary-pulsation-type devices (Fig. 2) have proven themselves well for homogenization, dispersion, etc. In these devices, there is an intense impact on the processed medium by acoustic impulse cavitations, hydraulic shocks, shear stresses, which leads to an increase in the efficiency of the processes being carried out. RPA differs from the extraction apparatus used at modern enterprises by low specific energy and metal consumption with high quality of the resulting finished product [12].

Extraction in RPA is based on the circulation and multiple processing of the medium in the internal volume of the apparatus. During RPA operation, large velocity gradients are observed, an intense mechanical effect on the particles of the raw material occurs, effective turbulization and flow pulsation occurs.

Excluding the grinding stage from the technological cycle. Under various operating modes of the rotary-pulsating extractors, after 70-90 seconds, the solid phase particles acquire a final size not exceeding $0.05 \mathrm{~mm}$ (with an inter-cylinder gap of $0.1 \mathrm{~mm}$ ). The phenomena accompanying the operation of these extractors contribute to a reduction in the duration of extraction, an increase in the yield of extracted substances, as well as an increase in the quality characteristics of the obtained extracts. 




Fig. 2. Rotary-pulsating apparatus with guide vanes: 1-body, 2-rotor, 3-holes, 4-stator, 5-cover, 6blades, 7-inlet, 8-outlet, 9-heat jacket, 10 - inlet heat jacket branch pipe, 11 - heat jacket outlet branch pipe, 12-inner working area, 13-rotor working surface, 14-outer working area.

RPA consists of a housing 1 with a rotor 2 installed in it, in the hub 3 of which there are holes, and a stator 4 made in the form of alternating coaxial cylinders with slots. The slotted stator is rigidly fixed to the cover 5 . In the area between the rotor teeth and the inner wall of the housing, rectangular blades 6 are located, bent along a helical line towards the rotor rotation. Connections 7 and 8 are for input and output of components. The body of the apparatus is equipped with a thermal jacket 9, with fittings 10 and 11 for supplying and removing the coolant. The device has working cavities 12 and 14, which are located between the wall and the teeth, as well as a working area 13 inside the rotor.

The device works as follows. Through the inlet 7, the components of the liquid and solid phases are fed into the working area 13, where, under the action of centrifugal forces, the flow moves through the slots of the teeth of the rotor 2 and the stator 4 . Then, in the working cavity of the apparatus 12, the flow is directed by rectangular blades 6 into the external cavity 14, where due to the pumping effect passes through the holes in the rotor hub 3. When the rotor 2 rotates, the slots of the rotor and stator overlap, as a result of which pulsations of the flow and its turbulization occur.

Due to the blades 6 fixed on the inner wall of the housing, an intense, directed and repeated circulation of the flow occurs in the outer cavity and the working area of the apparatus.

A similar apparatus, with an active hydrodynamic effect on the solid phase, is a centrifugal extractor, shown in Fig. 3 [12-13].

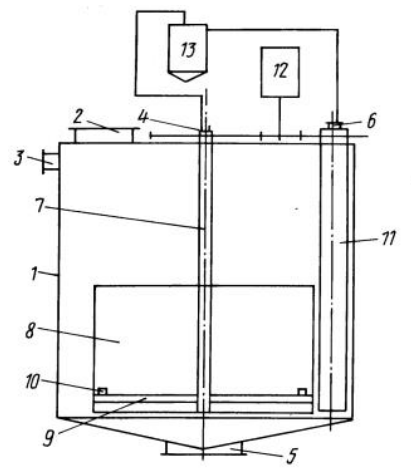

Fig. 3. Centrifugal extractor: 1-body, 2-6 nozzles, 7-hollow shaft, 8-mixer, 9-bubbler, 10-nozzle, 11filter element, 12-drive, 13-collection. 
The extractor contains a housing 1 with nozzles 2-6 for feedstock supply, extractant supply, extractant recirculation, feedstock withdrawal and solvent withdrawal, respectively, placed in housing 1 on a hollow shaft 7, a blade mixer 8 with a bubbler 9 equipped with supersonic nozzles 10, and a hollow filter element 11 , made in the form of a body of revolution, the cavity of which is in communication with the branch pipe 6 of the solvent outlet, as well as the rotation drive 12 connected with the shaft 7 and the filter element 11 , and the heated collector 13, located between the branch pipes of the solvent 6 and recirculation of the extractant 4 , the last of which communicated through the cavity of the shaft 7 with the bubbler 9 .

When the extractor is operating, the plant raw material is fed through the nozzle 2 to the housing 1 . The extractant is poured into the same nozzle 3 and the drive 12 is turned on. The rotation is transferred to the filter element 11 and through the shaft 7 to the mixer 8 and the bubbler 9. In the field of centrifugal forces, the extraction mixture is mixed and tangential filtration of the solvent passing through the filter element 11 into the branch pipe 6 of the solvent outlet. In the conditions of tangential filtration, clogging of the pores of the element 121 is excluded, which increases the reliability of the extractor. The suspension from the branch pipe 6 enters the heated collector 13, in which the extractant boils, separating from the extract, and is fed through the branch pipe 4 and the shaft cavity 7 into the bubbler 9. In the nozzles 10, the extractant vapor reaches supersonic velocity and with adiabatic expansion enter the extraction mixture. At the exit from the nozzles 10, a turbulent breakdown of the steam flow occurs, accompanied by the formation and collapse of cavitations cavities. As a result of adiabatic expansion and heat exchange with the extraction mixture, the vapor bubbles are cooled and condensed with the collapse of the cavitations cavities. As a result, ultrasonic frequency pressure fluctuations are generated in the extraction mixture, under the influence of which the cell membranes of the plant material are destroyed, which leads to a multiple increase in the contact surface of the phases and a drop in the diffusion resistance of the plant material. This provides an intensification of the extraction. After the completion of the extraction, the mixture is completely drained from the housing 1 with the drive 12 running, which is then turned off and removed from the housing 1 meal through the pipe 5. The extract isolated from the mixture is drained from the collector 13 . Then the extractor cycle is repeated.

The generally accepted methods for evaluating the quality of samples of alcoholic beverages were used within the research [12].

The main indicator for evaluating the effectiveness of the apparatuses used is the solid content. The content of solid compounds was measured by drying. The studies were performed using a thermogravimetric humidity analyzer MS-70. Devices of this type are based on the principle of thermogravimetric measurements, where samples with a known initial mass are dried, residues are weighed and the relative change in mass is determined with accuracy to four decimal places. After setting the accuracy and drying temperature, the sample weight and completion temperature are determined automatically. When the drying speed reaches the set value, the measurement stops automatically. The value of the solid content in the sample is calculated automatically by the formula: $\mathrm{SC}=(\mathrm{MW} / \mathrm{MD}) \times 100 \%$, where MW is the mass of the wet sample, MD is the mass of the dried sample.

It is not enough to evaluate only the physical and chemical parameters of the resulting tinctures, which is also true for evaluating other products of the food industry. Thus, tasting evaluation was performed to determine the parameters of taste, aroma and color. The quality of alcoholic beverages was assessed using well-known standard methods based on the results of the tasting panel score [13].

The results were mathematically processed using the method of multiple regression analysis to determine the effects of variable parameters on the quality of the resulting drink [14-15]. 


\section{Results and discussion}

To solve the set goal and objectives, a number of experiments were carried out. The study of the influence of the structure of plant raw materials and the efficiency of its extraction in devices of various designs was carried out with varying technological parameters. For the Soxlhet extractor, the volume of the feed material was changed. And for RPA and centrifugal extractor, the rotation frequency of the working bodies was changed. An integral characteristic of the effectiveness of the process is its duration. Therefore, the assessment was carried out with the same control time of the apparatus.

The obtained results of the extraction process in the Soxlhet extractor are presented in Table 1.

Table 1. Soxlhet extractor.

\begin{tabular}{|c|c|c|c|c|c|c|c|c|}
\hline \multirow[b]{2}{*}{ No. } & \multicolumn{2}{|c|}{ Encoded } & \multicolumn{6}{|c|}{ Numerically } \\
\hline & $\mathrm{X} 1$ & $\mathrm{X} 2$ & $\mathrm{~V}, \mathrm{~cm}^{3}$ & $\tau, \min$ & $\mathrm{C}, \%$ & $\begin{array}{c}\text { Aroma, } \\
\text { score }\end{array}$ & $\begin{array}{c}\text { Colour, } \\
\text { score }\end{array}$ & $\begin{array}{l}\text { Taste, } \\
\text { score }\end{array}$ \\
\hline \multicolumn{9}{|c|}{ Chaga mushroom } \\
\hline 1 & + & + & 186 & 15 & 3,6 & 3,6 & 3.5 & 3,6 \\
\hline 2 & + & - & 186 & 5 & 2,2 & 2,1 & 2,9 & 3,2 \\
\hline 3 & + & 0 & 186 & 10 & 3,1 & 2,9 & 3,3 & 3,4 \\
\hline 4 & 0 & + & 124 & 15 & 2,6 & 3,3 & 3,4 & 3,5 \\
\hline 5 & 0 & - & 124 & 5 & 1,7 & 2,1 & 3,1 & 3,2 \\
\hline 6 & 0 & 0 & 124 & 10 & 2,2 & 2,9 & 3,2 & 3,1 \\
\hline 7 & - & + & 62 & 15 & 2,1 & 3,2 & 3,2 & 3,3 \\
\hline 8 & - & - & 62 & 5 & 1 & 1,9 & 2.8 & 3 \\
\hline 9 & - & 0 & 62 & 10 & 1,6 & 2,6 & 3 & 3,1 \\
\hline \multicolumn{9}{|c|}{ Rose hips } \\
\hline 1 & + & + & 186 & 15 & 3,7 & 3,8 & 3.8 & 3,7 \\
\hline 2 & + & - & 186 & 5 & 2,4 & 2,5 & 3,4 & 3,4 \\
\hline 3 & + & 0 & 186 & 10 & 3,1 & 3,1 & 3,7 & 3,5 \\
\hline 4 & 0 & + & 124 & 15 & 2,7 & 3,7 & 3,6 & 3,6 \\
\hline 5 & 0 & - & 124 & 5 & 1,9 & 2,3 & 3,4 & 3,4 \\
\hline 6 & 0 & 0 & 124 & 10 & 2,3 & 2,9 & 3,5 & 3,5 \\
\hline 7 & - & + & 62 & 15 & 2,4 & 3,4 & 3,4 & 3,3 \\
\hline 8 & - & - & 62 & 5 & 1,3 & 2,4 & 2.9 & 2,9 \\
\hline 9 & - & 0 & 62 & 10 & 1,8 & 2,7 & 3,3 & 3 \\
\hline \multicolumn{9}{|c|}{ Penny root } \\
\hline 1 & + & + & 186 & 15 & 3,9 & 3,7 & 4.0 & 3,8 \\
\hline 2 & + & - & 186 & 5 & 2,4 & 2,8 & 3,4 & 3,4 \\
\hline 3 & + & 0 & 186 & 10 & 3,2 & 3,4 & 3,8 & 3,7 \\
\hline 4 & 0 & + & 124 & 15 & 2,7 & 3,6 & 3,7 & 3,6 \\
\hline 5 & 0 & - & 124 & 5 & 1,8 & 2,6 & 3,4 & 3,3 \\
\hline 6 & 0 & 0 & 124 & 10 & 2,3 & 3,2 & 3,6 & 3,5 \\
\hline 7 & - & + & 62 & 15 & 2,5 & 3,4 & 3,5 & 3,3 \\
\hline 8 & - & - & 62 & 5 & 1,2 & 2,7 & 3.0 & 3 \\
\hline 9 & - & 0 & 62 & 10 & 1,9 & 3 & 3,3 & 3,2 \\
\hline
\end{tabular}

The results show that the estimated dry matter content in the resulting tincture directly depends on the amount of raw materials loaded into the apparatus and the duration of the process. And organoleptic indicators directly depend on the content of dry substances.

After mathematical processing of the obtained experimental data, the dependences of the content of dry substances were revealed depending on the operating parameters of the extraction process in the Soxlhet extractor (Fig. 4) [14-15]. 


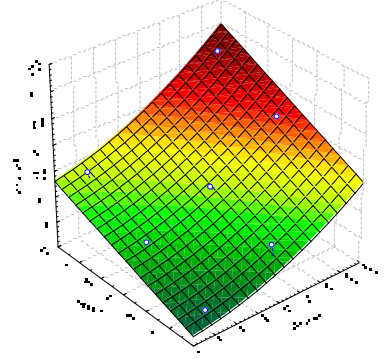

a

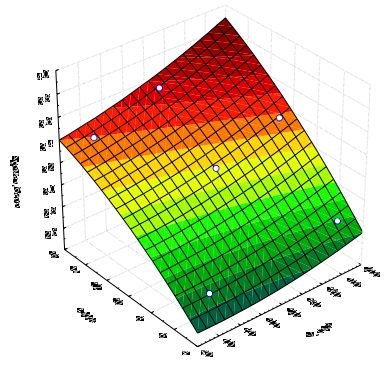

$\mathrm{b}$

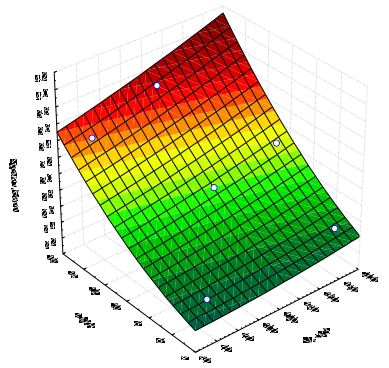

$\mathrm{C}$

Fig. 4. Response surfaces for the solid content in the Soxlhet extractor: $a-$ Rose hips, $b$ - Chaga mushroom, c - Penny root.

The results of the extraction process in a centrifugal extractor are presented in Table 2.

Table 2. Centrifugal extractor.

\begin{tabular}{|c|c|c|c|c|c|c|c|c|}
\hline \multirow{2}{*}{ No. } & \multicolumn{2}{|c|}{ Encoded } & \multicolumn{6}{|c|}{ Numerically } \\
\hline & $\mathrm{X} 1$ & $\mathbf{X} 2$ & $\mathrm{~V}, \mathbf{c m}^{\mathbf{3}}$ & $\tau, \min$ & $\mathrm{C}, \%$ & $\begin{array}{c}\text { Aroma, } \\
\text { score }\end{array}$ & $\begin{array}{c}\text { Colour, } \\
\text { score }\end{array}$ & $\begin{array}{l}\text { Taste, } \\
\text { score }\end{array}$ \\
\hline \multicolumn{9}{|c|}{ Chaga mushroom } \\
\hline 1 & + & + & 1600 & 15 & 3,9 & 3,5 & 3.6 & 3,5 \\
\hline 2 & + & - & 1600 & 5 & 2,5 & 2,2 & 3 & 3,2 \\
\hline 3 & + & 0 & 1600 & 10 & 3,3 & 2,9 & 3,3 & 3,4 \\
\hline 4 & 0 & + & 1400 & 15 & 3,8 & 3,4 & 3,4 & 3,3 \\
\hline 5 & 0 & - & 1400 & 5 & 2,5 & 2,4 & 3,2 & 3,1 \\
\hline 6 & 0 & 0 & 1400 & 10 & 3,2 & 3 & 3,3 & 3,2 \\
\hline 7 & - & + & 1200 & 15 & 2,9 & 3,2 & 3,3 & 3,3 \\
\hline 8 & - & - & 1200 & 5 & 2,2 & 2,2 & 2.9 & 3,2 \\
\hline 9 & - & 0 & 1200 & 10 & 2,7 & 2,7 & 3,1 & 3,2 \\
\hline \multicolumn{9}{|c|}{ Rose hips } \\
\hline 1 & + & + & 1600 & 15 & 4,2 & 3,8 & 3.8 & 3,6 \\
\hline 2 & + & - & 1600 & 5 & 2,8 & 2,5 & 3,4 & 3,4 \\
\hline 3 & + & 0 & 1600 & 10 & 3,7 & 3,1 & 3,6 & 3,5 \\
\hline 4 & 0 & + & 1400 & 15 & 3,9 & 3,7 & 3,5 & 3,5 \\
\hline 5 & 0 & - & 1400 & 5 & 2,9 & 2,3 & 3,4 & 3,4 \\
\hline 6 & 0 & 0 & 1400 & 10 & 3,5 & 2,9 & 3,2 & 3,5 \\
\hline 7 & - & + & 1200 & 15 & 3,1 & 3,4 & 3,3 & 3,5 \\
\hline 8 & - & - & 1200 & 5 & 2,2 & 2,4 & 2.9 & 3,3 \\
\hline 9 & - & 0 & 1200 & 10 & 2,8 & 2,7 & 3,3 & 3,3 \\
\hline \multicolumn{9}{|c|}{ Penny root } \\
\hline 1 & + & + & 1600 & 15 & 4,3 & 3,6 & 3.9 & 3,8 \\
\hline 2 & + & - & 1600 & 5 & 3 & 2,6 & 3,4 & 3,5 \\
\hline 3 & + & 0 & 1600 & 10 & 3,7 & 3,3 & 3,7 & 3,7 \\
\hline 4 & 0 & + & 1400 & 15 & 4,1 & 3,4 & 3,7 & 3,7 \\
\hline 5 & 0 & - & 1400 & 5 & 2,9 & 2,5 & 3,4 & 3,5 \\
\hline 6 & 0 & 0 & 1400 & 10 & 3,6 & 3 & 3,6 & 3,6 \\
\hline 7 & - & + & 1200 & 15 & 3,2 & 3,2 & 3,6 & 3,5 \\
\hline 8 & - & - & 1200 & 5 & 2,3 & 2,3 & 3.2 & 3,4 \\
\hline 9 & - & 0 & 1200 & 10 & 2,8 & 2,8 & 3,4 & 3,5 \\
\hline
\end{tabular}

Based on the results of experimental data, it can be concluded that the response of the dry matter content of the extract from rose hips, chaga mushroom and penny root is affected by the number of rotor revolutions, slightly less influenced by the extraction time. 
In each case, the best results are obtained at the number of revolutions from 1350 to 1500 . The organoleptic characteristics are significantly influenced by the rotor speed and to a slightly lesser extent the extraction time has an effect.

After mathematical processing of the obtained experimental data, the dependences of the content of dry substances were revealed depending on the operating parameters of the extraction process in a centrifugal extractor (Fig. 5) [14-15].

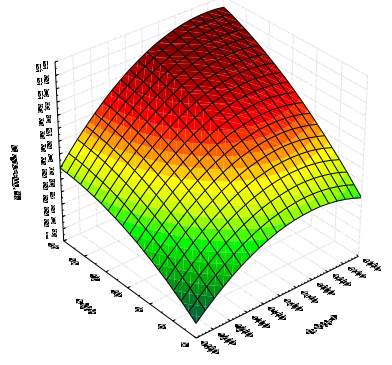

a

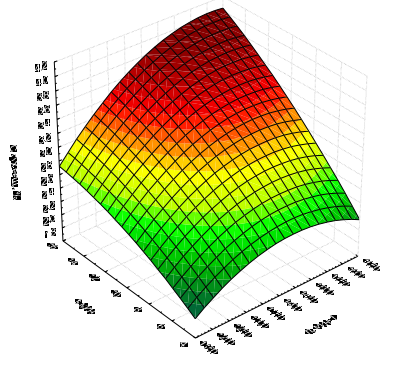

b

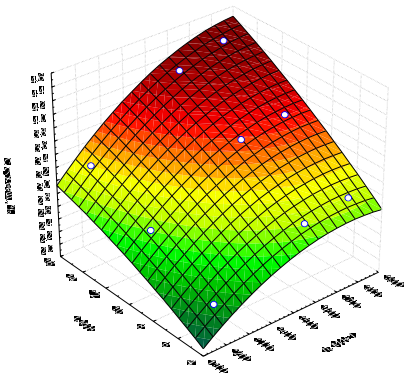

$\mathrm{c}$

Fig. 5. Response surfaces for the solid content in the Centrifugal extractor: $a$ - Rose hips, $b$ - Chaga mushroom, c - Penny root.

Experimental data of the extraction process in RPA are presented in Table 3.

Table 3. Rotary-pulsating apparatus.

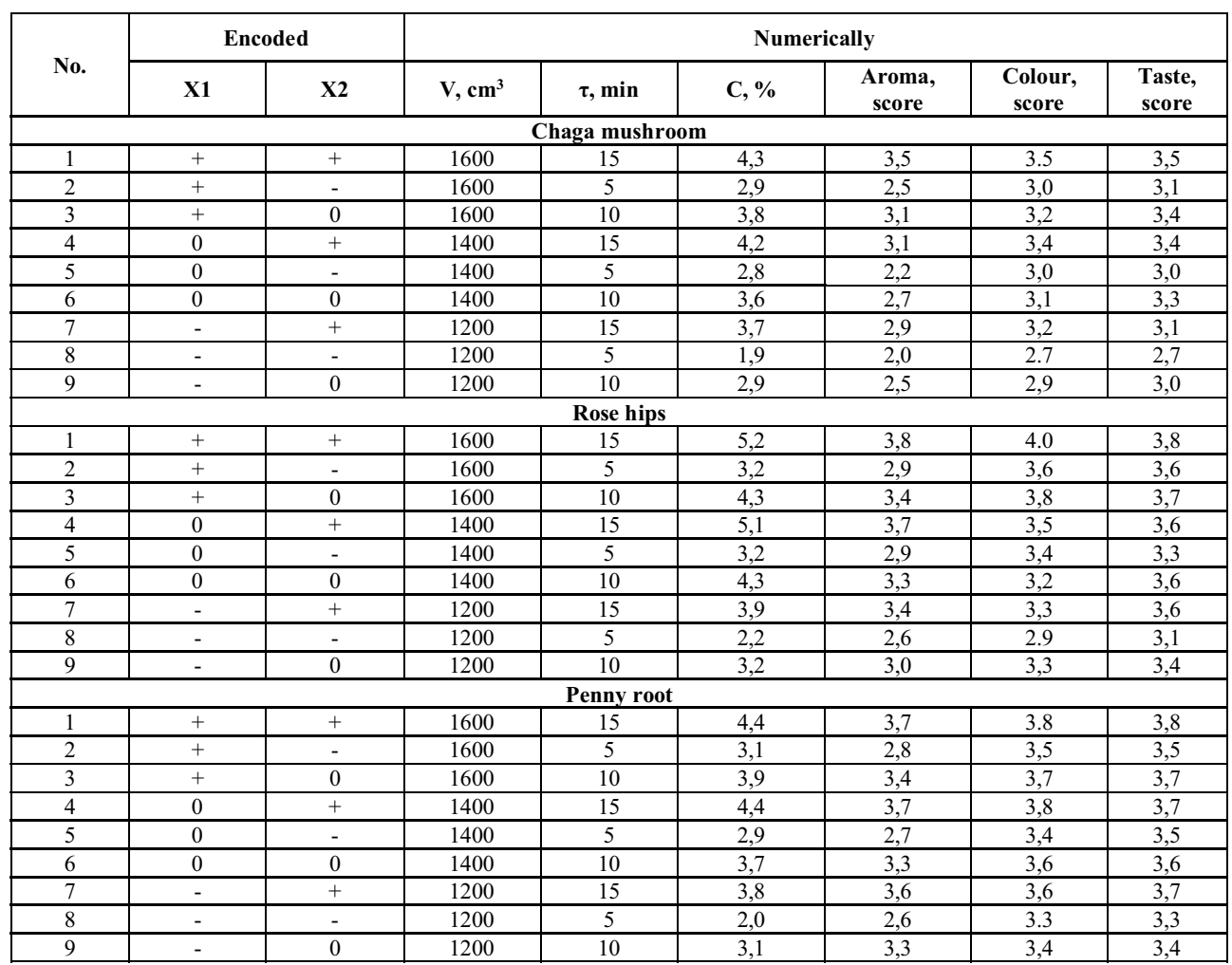


From the data obtained, it can be seen that the dependence of the content of dry matter in the extract from rose hips, chaga mushroom and penny root is influenced by the number of rotor revolutions. The extraction time has a lesser effect. High indicators for the yield of dry substances from raw materials are achieved due to the fact that RPA has disintegrating properties. Raw materials of any structure undergo hydromechanical processing, which results in an increased yield of dry substances.

After mathematical processing of the obtained experimental data, the dependences of the content of dry substances were revealed depending on the operating parameters of the extraction process in a centrifugal extractor (Fig. 6) [14-15].



a



b

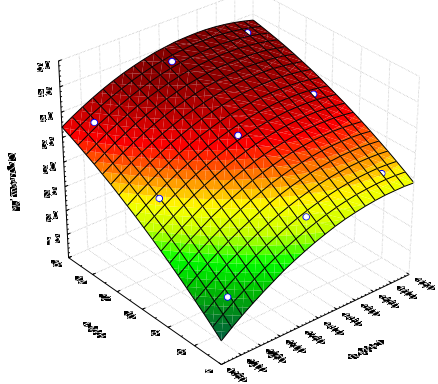

c

Fig. 6. Response surfaces for the solid content in the RPA: a - Rose hips, b - Chaga mushroom, c Penny root.

The experimental results showed that the most effective extractor for extracting biologically active substances from the roots and rhizomes of plant raw materials is Soxlhet extractor. Due to the thermal effect in the extractor, alcohol vapors freely penetrate into the structure of the roots, which have a high conductive capacity of nutrients and minerals. Determined rational technological parameters of work for the Soxlhet extractor for all types of raw materials: $\mathrm{V}=170 \mathrm{~cm}^{3}$ and time $=12 \mathrm{~min}$ (Chaga mushroom), $\mathrm{V}=165 \mathrm{~cm}^{3}$ and time $=14 \mathrm{~min}$ (Rose hips), $\mathrm{V}=164 \mathrm{~cm}^{3}$ and time $=12 \mathrm{~min}$ (Penny root).

Due to the fact that fruit and berry raw materials and rose hips are small in size with a loose structure, centrifugal apparatus must be used for the most efficient extraction process, because they have the ability to crush the raw material with its simultaneous mechanical processing, which significantly reduces the preparation time of the extract. Determined rational technological parameters of work for a centrifugal extractor with all types of raw materials: $\mathrm{n}=1550 \mathrm{~min}^{-1}$ and $\tau=14 \mathrm{~min}$ (Chaga mushroom), $\mathrm{n}=1550 \mathrm{~min}^{-1}$ and $\tau=14$ $\min$ (Penny root), $\mathrm{n}=1400 \mathrm{~min}^{-1}$ and $\tau=11$ min (Rose hips).

Since the chaga mushroom has a large amount of bark in its structure, the preparation of extracts requires either preliminary preparation or the use of an apparatus capable of crushing such raw materials. The rotor-pulsating apparatus perfectly coped with this task, because it is in it that the necessary hydrodynamic effects are created (pulsation, turbulization and the effect of cavitations forces) with simultaneous mechanical action. Determined rational technological parameters of work for a rotary-pulsating apparatus for all types of raw materials: $\mathrm{n}=1550 \mathrm{~min}^{-1}$ and $\tau=12 \mathrm{~min}$ (Chaga mushroom), $\mathrm{n}=1450 \mathrm{~min}^{-}$ ${ }^{1}$ and $\tau=12 \min$ (Penny root), $\mathrm{n}=1400 \mathrm{~min}^{-1}$ and $\tau=10 \mathrm{~min}$ (Rose hips). 


\section{Conclusions}

The research carried out makes it possible to evaluate the efficiency of the considered designs of extractors in the "solid - liquid" system. Depending on the structure of plant materials, the type of apparatus also changes.

The Soxlhet extractor shows high efficiency on raw materials with large pores in the structure. Alcohol vapors penetrate deeply into the material and extract the most completely. But the duration of the process increases. Of the most important advantages of this apparatus, a significantly smaller amount of solvent is required. Since its constant circulation occurs throughout the entire volume of the apparatus.

The centrifugal extractor and the rotary-pulsating apparatus show great efficiency, since they have a hydromechanical effect on any raw material.

The most versatile apparatus is the rotary-pulsating apparatus, since during its operation the raw material is crushed to a size of $0.01 \mathrm{~mm}$. As a result, plant raw materials give the most complete target components with little time.

Reducing production costs and production costs is an urgent task for organizations in all industries. This becomes achievable only with the right selection of equipment. Therefore, based on the manufactured products, it is necessary to qualitatively study the market and designs of the proposed devices. Then choose exactly the one that has shown its effectiveness with the processed raw materials. Or choose universal devices.

\section{References}

1. D. Borodulin, Storage and Processing of Farm Products, 4, 141-453 (2019), DOI: https://doi.org/10.36107/spfp.2019.154

2. B. Fedorenko, Food Processing: Techniques and Technology, 50(1), 115-123 (2020), DOI: https://doi.org/10.21603/2074-9414-2020-1-115-123

3. L. Dyshlyuk, Journal of Food Process Engineering, 40(1), 54-67 (2017), DOI: https://doi.org/10.1111/jfpe.12331

4. L. Gnetko, International Journal of Innovative Technology and Exploring Engineering, 8 (9), 1978-1981 (2019), DOI: https://doi.org/10.35940/ijitee.i8317.078919

5. S. Kodama, Chemical Engineering and Processing: Process Intensification, 97, 45-54 (2015), DOI: https://doi.org/10.1016/j.cep.2015.08.012

6. N. More, Chemical Engineering and Processing: Process Intensification, 128, 132-142 (2018), DOI: https://doi.org/10.1016/j.cep.2018.04.008

7. D. Pradal, Chemical Engineering and Processing: Process Intensification, 127, 83-92 (2018), DOI: https://doi.org/10.1016/j.cep.2018.03.016

8. Z. Tazova, International Journal of Engineering and Advanced Technology (IJEAT), 9(1) (2019), DOI: https://doi.org/10.35940/ijeat.A1772.109119

9. M. Prosin, Processes and Food Production Equipment, 4, 3-12 (2017), DOI: https://doi.org/10.17586/2310-1164-2017-10-4-3-12

10. D. Borodulin, E3S Web Conf., 175, 08010 (2020), DOI: https://doi.org/10.1051/e3sconf/202017508010

11. E. Safonova, Food Processing: Techniques and Technology, 50 (4), 630-641 (2020), DOI: https://doi.org/10.21603/2074-9414-2020-4-630-641

12. K. Hurkova, Food Chemistry, 284, 162-170 (2019), DOI: https://doi.org/10.1016/j.foodchem.2019.01.014 
13. R. Evangelista, Industrial Crops and Products, 29(1), 189-196 (2009), DOI: https://doi.org/10.1016/j.indcrop.2008.04.024

14. G. Rudoy, Pattern Recognition and Image Analysis, 26(23), 608-616 (2016), DOI: https://doi.org/10.1134/S1054661816030172

15. J. Wu, Journal of Electronics and Information Technology, 40(5), 1195-1201 (2018), DOI: https://doi.org/10.11999/JEIT170624

16. A. Potapov, Food Processing: Techniques and Technology, 3, 80-84 (2013) 